\title{
Avaliação dos Comportamentos Mecânico e Térmico de Laminados de PPS/Fibra de Carbono Processados em Autoclave sob Diferentes Ciclos de Consolidação
}

\author{
Luciana S. Marques, Nilson E. Narita, Gustavo G. da Costa \\ Engenharia Aeronáutica e Mecânica, ITA \\ Mirabel C. Rezende \\ Instituto Tecnológico de Aeronáutica, IAE
}

\begin{abstract}
Resumo: Usualmente, um dos processos mais utilizados para fabricação de componentes em termoplásticos estruturais é a moldagem por compressão a quente, porém restringindo-se na obtenção de peças de pequeno e médio porte. Tal restrição deve-se à limitação do tamanho das prensas utilizadas, principalmente pelo custo envolvido. Procurando ampliar a aplicação de compósitos termoplásticos, possibilitando a fabricação de peças maiores e com maior potencial de integração, pelo uso de infra-estrutura já disponível em processadores de compósitos, este trabalho aborda o processamento de laminados de poli(sulfeto de fenileno) (PPS) reforçado com fibra de carbono em autoclave, pelo uso de quatro diferentes ciclos de consolidação. Os laminados obtidos foram caracterizados por inspeção por ultrassom, análises de DSC para a determinação da cristalinidade e avaliação das propriedades mecânicas em flexão, compressão e cisalhamento interlaminar. Os resultados mostram que laminados obtidos com taxas de resfriamento mais lentas apresentam menor resistência e módulo em compressão, uma vez que o maior grau de cristalinidade ( 30\%) promove maior fragilização da matriz polimérica.
\end{abstract}

Palavras-chave: Compósito termoplástico, PPS, poli(sulfeto de fenileno), consolidação em autoclave.

\section{Evaluation of Thermal and Mechanical Behaviors of PPS/Carbon Fiber Laminates Processed in Autoclave Under Different Consolidation Cycles}

Abstract: One of the most used processes to obtain structural thermoplastic parts is the hot compression molding, which is limited to small and medium size parts because of the size of the press used, mainly related to its cost. This has hampered a wide use of thermoplastic composites in structural applications. In order to extend the application of thermoplastic composites, but still using available infrastructure in composite manufacturers, in this work we investigate the processing of carbon fiber reinforced polyphenylene sulfide (PPS) in an autoclave, using four consolidation cycles. The processed laminates were characterized by ultrasound inspection, DSC analyses for the crystallinity determination and mechanical tests to evaluate the compression, flexure and interlaminar shear strength (ILSS). The results show that the laminates obtained under the lowest cooling rate exhibited the lowest compressive strength and modulus, because of the higher crystallinity degree $(\sim 30 \%)$ that turns the matrix more brittle.

Keywords: Thermoplastic composite, PPS, polyphenylene sulfide, consolidation in autoclave.

\section{Introdução}

$\mathrm{Na}$ indústria aeronáutica, os materiais compósitos poliméricos surgem como uma alternativa às estruturas metálicas tradicionalmente utilizadas desde o início da aviação. Os materiais compósitos, com suas variadas combinações de matriz e reforço, conferem à estrutura menor peso em relação às estruturas metálicas, consequentemente, maiores resistência mecânica e rigidez específicas. A obtenção de componentes com esse tipo de material possibilita, ainda, uma maior integração de peças, um menor custo de fabricação e maiores resistências à corrosão, a ataques químicos e às intempéries. Seu uso, inicialmente restrito a área de mísseis e foguetes, tem se tornado cada vez mais frequente em diferentes áreas da engenharia. Nesse sentido, estima-se um aumento de $5 \%$ ao ano na utilização de compósitos poliméricos estruturais, também denominados avançados ${ }^{[1]}$. E, projeções para a próxima década apontam o uso de 35 a $80 \%$ em massa de materiais compósitos nos diferentes setores da indústria aeronáutica ${ }^{[2]}$.

Dentre as matrizes poliméricas mais utilizadas no processamento de compósitos de uso na indústria aeronáutica encontram-se as termorrígidas, como as resinas fenólicas, epoxídicas e bismaleimidas. No entanto, tem-se observado, mais recentemente, a utilização crescente de matrizes termoplásticas, como o poli(sulfeto de fenileno) (PPS), a poli(éter-imida) (PEI), a poli(éter-éter-cetona) (PEEK) e a poli(éter-cetona-cetona) (PEKK). Compósitos à base de PEI, por exemplo, têm sido empregados em peças de interior de aeronaves, por apresentarem excelente desempenho quanto à inflamabilidade e boas propriedades mecânicas ${ }^{[3,4]}$. Já compósitos de matrizes de PPS, PEEK e PEKK são requisitados para aplicações que exigem elevado desempenho estrutural, pois esses polímeros conferem ao material compósito melhores propriedades mecânicas. Os polímeros PEEK e PEKK destacam-se ainda mais por manterem suas elevadas rigidez em altas temperaturas (temperatura de transição vítrea $\left(\mathrm{T}_{\mathrm{g}}\right)$ em torno de $\left.150^{\circ} \mathrm{C}\right)^{[5,6]}$.

Nesse sentido, a matriz de PPS vem também se destacando pelo seu processamento ocorrer em temperaturas menores que as necessárias para o PEEK e PEKK, e por conferir boas propriedades mecânicas a laminados com requisitos de uso em temperaturas mais altas $\left(\mathrm{T}_{\mathrm{g}} \text { PPS puro }=89^{\circ} \mathrm{C} \text { e Tg PPS em compósitos }=126^{\circ} \mathrm{C}\right)^{[7-9]}$. A Tabela 1 apresenta valores de resistências à compressão e flexão 
de laminados de PPS/fibras de carbono ${ }^{[7]}$, onde se observam as atrativas propriedades apresentadas por esse material.

A maioria dos termoplásticos de alto desempenho apresentam morfologia semicristalina, com no máximo cerca de $90 \%$ de cristalinidade, uma vez que não é possível obter polímeros $100 \%$ cristalino. A cristalinidade influencia significativamente nas propriedades químicas e mecânicas desses materiais, sendo que em geral, a cristalinidade tende a aumentar a resistência à tração, a rigidez e a resistência a solventes, enquanto que a fase amorfa confere a característica de maior absorção da energia de impacto ${ }^{[5,8]}$.

O grau de cristalinidade é consequência de diversos fatores, incluindo a natureza do polímero e suas condições de processamento. Durante o processamento de um termoplástico semicristalino, os cristais da fase cristalina se formam no período de resfriamento da fase fundida. Assim, a taxa de resfriamento é o parâmetro de maior influência na determinação da cristalinidade do polímero ${ }^{[5,10]}$.

O uso de compósitos termoplásticos na indústria aeronáutica torna-se mais comum à medida que o custo desses materiais é reduzido. O elevado custo dos compósitos termoplásticos está associado ao maior custo da matéria-prima, mas, principalmente, ao elevado custo de processamento, diretamente afetado pelo processo de fabricação utilizado ${ }^{[11]}$. Sendo assim, a maior utilização de compósitos termoplásticos está diretamente condicionada à redução dos custos de fabricação, consequentemente, à utilização de um processo otimizado.

Os custos dos processos de fabricação, no caso dos compósitos termoplásticos, estão atrelados, basicamente, à sua natureza térmica. Processos isotérmicos, onde o material é aquecido através do ferramental e do ambiente ao redor, como por exemplo, consolidação em autoclave, o custo é fortemente dependente do ciclo térmico nos valores de temperatura máxima. Já processos não isotérmicos, onde o material é aquecido diretamente, os custos de fabricação derivam dos custos direto e indireto de mão-de-obra ${ }^{[11]}$.

Considerando o excelente potencial de aplicação dos compósitos termoplásticos em componentes estruturais, a necessidade de se adequar as técnicas de processamento na obtenção de componentes de maior porte e a influência da cristalização de matrizes semicristalinas no desempenho mecânico do material processado ${ }^{[10]}$, este trabalho foi realizado visando a adequação do processamento de laminados de PPS/fibra de carbono, garantindo as boas propriedades mecânicas desses compósitos termoplásticos. Para isto, buscou-se otimizar o uso de infra-estrutura já disponível e normalmente utilizada para o processamento de compósitos termorrígidos, ou seja, a utilização de autoclave, em substituição, por exemplo, à moldagem por compressão a quente.

O processo de obtenção de compósitos termoplásticos por consolidação em autoclave é atualmente utilizado por grandes fabricantes aeronáuticos, como por exemplo a empresa Airbus, na fabricação do bordo de ataque da aeronave A340-600 e a empresa Gulfstream na fabricação do leme e profundor da aeronave G650. Ainda nesse sentido, outros estudos se apresentam para contribuir no desenvolvimento dessa tecnologia ${ }^{[12,13]}$, na avaliação mais detalhada do comportamento mecânico desses materiais, bem como no estudo da influência da temperatura nas características finais dessa classe de compósitos poliméricos ${ }^{[8,14,15]}$.

Nesse sentido, este presente estudo mostra o trabalho realizado no processamento de compósitos de poli(sulfeto de fenileno) reforçados com tecido de fibra de carbono em autoclave, sob diferentes ciclos de consolidação. Os diferentes laminados obtidos foram avaliados quanto às suas propriedades mecânicas em compressão, flexão e cisalhamento interlaminar e também quanto aos seus comportamentos térmicos.

\section{Experimental}

\section{Materiais}

Foi utilizado neste estudo tecido de fibra de carbono, com tecelagem tipo cetim (T300 3K 5HS), semi-impregnado em ambos os lados com filme de PPS (semipreg), fornecido pela empresa holandesa Ten Cate Advanced Composites, sob a sigla CD0286.

\section{Processamento}

Para a obtenção das amostras de compósitos, oito camadas de semipreg foram laminadas manualmente sobre um ferramental de aço, que foi preparado com desmoldante líquido para uso na temperatura de processamento. Ao redor das camadas foi disposto tecidos de fibra de vidro, que serviu como canalizador, auxiliando na retirada do ar aprisionado entre as camadas de semipreg. Sobre o semipreg foi utilizado filme separador e, em seguida, todo o conjunto foi isolado em uma bolsa de vácuo resistente a $320^{\circ} \mathrm{C}$. Os laminados foram consolidados em autoclave sob quatro diferentes ciclos de temperatura e pressão, resultando, portanto, em quatro famílias de laminados. Os diferentes ciclos de consolidação realizados neste trabalho estão apresentados na Tabela 2.

As células sombreadas na Tabela 2 mostram os diferentes parâmetros utilizados, quando comparados com os do ciclo de consolidação de referência, Ciclo $\mathrm{C}$, que é o ciclo de processamento recomendado pelo fornecedor da matéria-prima. Nesse sentido, o Ciclo A difere do Ciclo C por não apresentar pressão durante o aquecimento. O Ciclo $\mathrm{B}$, por sua vez, além de não apresentar pressão durante o aquecimento, tem menores taxas de aquecimento e resfriamento e o Ciclo $\mathrm{D}$, além de não ter pressão durante o aquecimento, usa maior pressão durante o resfriamento, quando comparado com o Ciclo C.

Os laminados obtidos em cada ciclo de consolidação foram caracterizados por inspeção não destrutiva pela técnica de ultrassom, por ensaios de resistência mecânica em compressão, flexão e cisalhamento interlaminar e, também, com relação aos seus comportamentos térmicos, via análises por calorimetria exploratória diferencial (DSC).

Tabela 1. Propriedades mecânicas de laminados de PPS/fibra de carbono ${ }^{[7]}$.

\begin{tabular}{ccccc}
\hline $\begin{array}{c}\text { Direção do } \\
\text { reforço/ } \\
\text { ensaio }\end{array}$ & $\begin{array}{c}\text { Resistência } \\
\text { à flexão } \\
\text { (MPa) }\end{array}$ & $\begin{array}{c}\text { Módulo } \\
\text { em flexão } \\
\text { (GPa) }\end{array}$ & $\begin{array}{c}\text { Resistência à } \\
\text { compressão } \\
\text { (MPa) }\end{array}$ & $\begin{array}{c}\text { Módulo em } \\
\text { compressão } \\
\text { (GPa) }\end{array}$ \\
\hline $0^{\circ}$ & 1027 & 60 & 644 & 51 \\
$90^{\circ}$ & 831 & 45 & 637 & 51 \\
\hline
\end{tabular}

Tabela 2. Ciclos de consolidação dos semipregs de PPS/fibra de carbono realizados em autoclave.

\begin{tabular}{|c|c|c|c|c|c|}
\hline \multicolumn{2}{|c|}{ Parâmetros } & \multicolumn{4}{|c|}{ Ciclos de consolidação } \\
\hline & & $\mathbf{A}$ & B & $\mathbf{C}$ & D \\
\hline \multirow[t]{2}{*}{ Aquecimento } & $\begin{array}{c}\text { Taxa } \\
\left({ }^{\circ} \mathrm{C} / \mathrm{min}\right)\end{array}$ & $10-20$ & $1-5$ & $10-20$ & $10-20$ \\
\hline & $\begin{array}{l}\text { Pressão } \\
(\mathrm{kPa})\end{array}$ & 0 & 0 & $400-600$ & 0 \\
\hline \multirow[t]{3}{*}{ Patamar } & $\begin{array}{c}\text { Temperatura } \\
\left({ }^{\circ} \mathrm{C}\right)\end{array}$ & $300-315$ & $300-315$ & $300-315$ & $300-315$ \\
\hline & $\begin{array}{c}\text { Tempo } \\
\text { (minutos) }\end{array}$ & $20-60$ & $20-60$ & $20-60$ & $20-60$ \\
\hline & $\begin{array}{l}\text { Pressão } \\
(\mathrm{kPa})\end{array}$ & $600-900$ & $600-900$ & $600-900$ & $600-900$ \\
\hline \multirow[t]{2}{*}{ Resfriamento } & $\begin{array}{c}\text { Taxa } \\
\left({ }^{\circ} \mathrm{C} / \mathrm{min}\right)\end{array}$ & $10-20$ & $1-5$ & $10-20$ & $10-20$ \\
\hline & $\begin{array}{c}\text { Pressão } \\
(\mathrm{kPa})\end{array}$ & $600-900$ & $600-900$ & $600-900$ & $900-1200$ \\
\hline
\end{tabular}




\section{Inspeção por ultrassom}

Para avaliar a qualidade dos laminados fabricados foram realizadas inspeções pela técnica de ultrassom. O método utilizado foi o $C$-Scan por transmissão via imersão em água, que permite a verificação de possíveis descontinuidades no compósito, como por exemplo, regiões ricas em resina e vazios. Para isso, neste trabalho foi utilizada uma placa denominada "padrão termoplástico", como referência na comparação com as quatro famílias de laminados fabricadas. Esse padrão foi obtido pelo mesmo processo de consolidação em autoclave, através do Ciclo C, uma vez que esse é o ciclo recomendado pelo fornecedor da matéria-prima ${ }^{[10]}$. Ensaios adicionais, como conteúdo de resina e fibra, porcentagem de vazios e análises por estereoscopia foram realizados para certificar sua qualidade, garantindo que essa placa de referência encontrava-se isenta de descontinuidades.

\section{Análise por calorimetria exploratória diferencial (DSC)}

Os laminados, após a consolidação, foram analisados em triplicata, utilizando um equipamento de DSC da Perkin Elmer, modelo Pyris 1, sob fluxo constante de $\mathrm{N}_{2}(20 \mathrm{~mL} / \mathrm{min})$, previamente calibrado com padrões de índio e zinco. As amostras, encapsuladas em porta-amostras de alumínio, foram aquecidas a $11^{\circ} \mathrm{C} / \mathrm{min}$ até $315{ }^{\circ} \mathrm{C}$, onde permaneceram por 2 minutos para garantir a fusão de toda a matriz polimérica. A partir das curvas geradas no primeiro aquecimento foram obtidos a entalpia de fusão e o grau de cristalinidade de cada amostra, sendo esse último parâmetro calculado conforme a Equação 1:

$$
X_{C}=\frac{\Delta H_{f}+\Delta H_{c}}{\left(1-W_{f}\right) \Delta H_{100 \%}}
$$

onde: $\mathrm{X}_{\mathrm{c}}$ é o grau de cristalinidade (em \%), $\Delta \mathrm{H}_{\mathrm{f}}$ é a entalpia de fusão, $\Delta \mathrm{H}_{\mathrm{c}}$ é a entalpia de cristalização no aquecimento, também denominada de cristalização a frio ${ }^{[6]}, \Delta \mathrm{H}_{100 \%}$ é a entalpia de fusão para o PPS $100 \%$ cristalino e $\mathrm{W}_{\mathrm{f}}$ é a fração em massa do reforço do compósito. Neste trabalho, o valor para $\Delta \mathrm{H}_{100 \%}$ utilizado foi

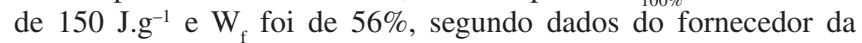
matéria-prima, a empresa Ten Cate ${ }^{[10]}$.

\section{Ensaios mecânicos}

A caracterização mecânica dos laminados processados foi realizada por meio de ensaios de compressão, flexão e cisalhamento interlaminar, em seis corpos de prova cada. Esses ensaios foram realizados em uma máquina de ensaios universal da MTS, modelo 10T.743.D. Os ensaios de compressão foram conduzidos de acordo com os procedimentos da norma ASTM D 6641 para a determinação da resistência, com um deslocamento de $1,3 \mathrm{~mm} / \mathrm{min}$ para obtenção da resistência. Para a obtenção do módulo em compressão, os ensaios foram baseados nos procedimentos da norma SACMA SRM1-94.

Para a obtenção da resistência ao cisalhamento interlaminar, os corpos de prova foram ensaiados conforme a norma ASTM D 2344.

Os ensaios de flexão foram conduzidos segundo a norma européia EN 2562, tipo B e utilizados para determinar o módulo elástico e a resistência na ruptura dos laminados produzidos.

\section{Resultados e Discussão}

\section{Inspeção por ultrassom}

A Figura 1 apresenta as imagens obtidas na inspeção por ultrassom, realizada nos laminados fabricados com diferentes parâmetros dos ciclos de consolidação (Tabela 2) e na placa denominada "padrão termoplástico".
A análise das imagens da Figura 1 mostra que todos os laminados inspecionados não apresentam atenuação significativa do sinal de ultrassom, com valores praticamente equivalentes aos apresentados pelo laminado de referência. Este resultado indica boa distribuição dos componentes (reforço e matriz polimérica) nos laminados processados nos diferentes ciclos de consolidação. $\mathrm{Na}$ análise comparativa das imagens da Figura 1 também não se observa a presença de defeitos, como vazios, regiões ricas ou pobres em polímero, delaminações ou contaminações.

\section{Análise por calorimetria exploratória diferencial}

A Figura 2 apresenta, comparativamente, as curvas obtidas por DSC, que mostram o comportamento térmico apresentado pelos diferentes laminados processados neste estudo. Os picos dos eventos endotérmicos estão representados de forma ascendente e os picos exotérmicos são os descendentes. As análises foram realizadas a partir da primeira varredura, primeiro aquecimento, portanto representam a cristalinidade presente nos laminados processados sob os diferentes ciclos de consolidação.

As curvas obtidas nas análises de DSC para os laminados consolidados não apresentam a inflexão que define a $\mathrm{T}_{\mathrm{g}}$, em torno de $89{ }^{\circ} \mathrm{C}$, para o caso da matriz de PPS. Esse comportamento térmico não é evidenciado nas análises provavelmente pela discreta contribuição da região amorfa, associada aos parâmetros de análise utilizados. As referidas curvas também não mostram a presença de cristalização no aquecimento, representada por picos exotérmicos por volta de $120^{\circ} \mathrm{C}$, conforme menciona a literatura ${ }^{[6]}$. De acordo com a literatura, essa última evidência sugere a presença das regiões cristalinas bem formadas nas amostras obtidas nos diferentes ciclos de processamento ${ }^{[10,16]}$. A Tabela 3 apresenta as médias do grau de cristalinidade determinadas para as quatro famílias de laminados consolidados, conforme a Equação 1.

Os resultados da Tabela 3 mostram uma diferença significativa nos graus de cristalinidade determinados para os laminados obtidos pelo ciclo B de consolidação $(29,7 \%)$ e os demais, A, C e D $(18,1$ a $20,4 \%)$, que apresentam menores graus de cristalinidade. O ciclo $\mathrm{B}$, que resultou no laminado de maior grau de cristalinidade,

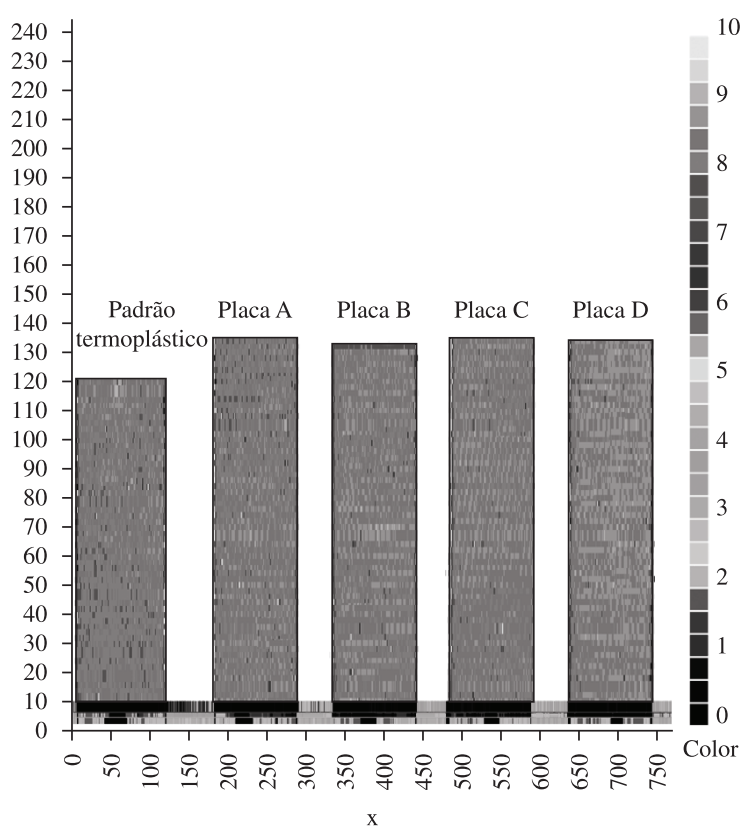

Figura 1. Imagens da inspeção por ultrassom dos laminados de PPS/fibra de carbono processados. 
refere-se ao ciclo de processamento que possui as menores taxas de aquecimento e resfriamento (Tabela 2). Assim, o comportamento observado é esperado, uma vez que resfriamentos mais rápidos de polímeros semicristalinos fundidos geralmente resultam em menor cristalinidade da matriz polimérica ${ }^{[9,10,17]}$.

Os resultados de cristalinidade determinados estão em concordância com os apresentados na literatura, em torno de $21 \%$, também obtidos experimentalmente para laminados de PPS/fibras de carbono, com taxa de resfriamento de $10{ }^{\circ} \mathrm{C} / \mathrm{min}^{[18]}$.

\section{Ensaios mecânicos}

As Figuras 3 e 4 mostram os resultados obtidos nos ensaios de compressão, em termos de resistência e módulo, respectivamente. Observa-se que, o laminado fabricado sob o ciclo de consolidação $\mathrm{B}$, com menores taxas de aquecimento e resfriamento em relação aos outros ciclos, apresenta menor resistência à compressão. Essa redução é de aproximadamente $12 \%$, quando comparada com a determinada para o laminado consolidado pelo ciclo $\mathrm{C}$, que apresenta o maior valor de resistência. Conforme sugere a literatura, menores taxas de resfriamento podem resultar em estruturas cristalinas melhor formadas e maiores, característica essa que induz, consequentemente, a um maior encolhimento volumétrico da matriz. Esse comportamento pode acarretar na formação de concentradores de tensão, que podem levar à uma maior fragilização do polímero termoplástico semicristalino ${ }^{[9,10]}$. No presente estudo, os resultados de resistência à compressão evidenciam esse comportamento, sabendo-se que o ensaio de compressão é influenciado pelas características da matriz.

A comparação dos resultados dos ensaios de resistência à compressão dos laminados processados em autoclave neste estudo, com dados do fabricante (Tabela 1) ${ }^{[7]}$, obtidos por moldagem por

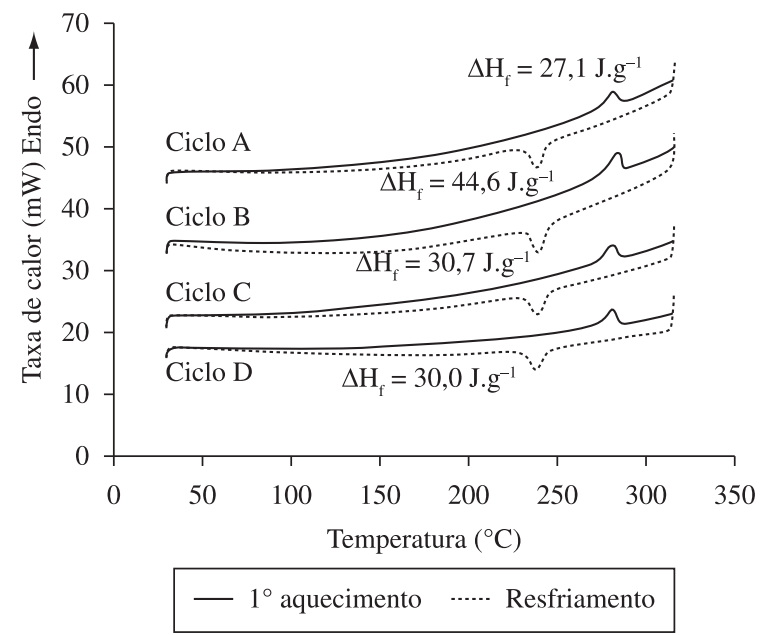

Figura 2. Curvas de DSC dos laminados PPS/fibra de carbono consolidados.

Tabela 3. Entalpias de fusão e graus de cristalinidade obtidos por DSC para as amostras de laminados de PPS/fibra de carbono.

\begin{tabular}{ccccc}
\hline $\begin{array}{c}\text { Parâmetros } \\
\text { DSC }\end{array}$ & \multicolumn{4}{c}{$\begin{array}{c}\text { Laminados conforme ciclos de consolidação } \\
\text { (valores médios) }\end{array}$} \\
\cline { 2 - 5 } & $\mathbf{A}$ & $\mathbf{B}$ & $\mathbf{C}$ & $\mathbf{D}$ \\
\hline$\Delta \mathrm{H}_{\mathrm{C}}\left(\mathrm{J}_{\mathrm{g}} \mathrm{g}^{-1}\right)$ & 0 & 0 & 0 & 0 \\
$\Delta \mathrm{H}_{\mathrm{f}}\left(\mathrm{J} \cdot \mathrm{g}^{-1}\right)$ & 27,1 & 44,6 & 30,7 & 30,0 \\
$($ Desv pad) & $(1,3)$ & $(1,3)$ & $(1,2)$ & $(1,0)$ \\
$\mathrm{X}_{\mathrm{C}}(\%)$ & 18,1 & 29,7 & 20,4 & 19,9 \\
\hline
\end{tabular}

compressão a quente, mostra boa concordância. Observa-se ainda que, os resultados oriundos dos ensaios das amostras do ciclo B apresentam maior dispersão dos dados, em relação às outras famílias ensaiadas, sugerindo uma menor homogeneidade da morfologia da matriz polimérica e/ou na interface reforço/polímero, talvez pela presença de regiões com diferentes graus de ordenamento cristalográfico da matriz.

Em relação aos resultados obtidos para o módulo em compressão, nenhuma diferença significativa é observada entre os laminados, sendo o maior desvio determinado de, aproximadamente, $2 \%$ entre as médias encontradas, e de $8 \%$ considerando os valores individuais das quatro famílias ensaiadas. Os valores médios de módulo determinados para os laminados ensaiados encontram-se em torno de $53 \mathrm{GPa}$. Comparando-se esses resultados com o disponibilizado pelo fabricante da matéria-prima $(51 \mathrm{GPa})^{[7]}$ e com dados da literatura, referentes a compósitos similares (PPS/fibra de carbono) obtidos por moldagem por compressão a quente $(54 \mathrm{GPa})^{[18]}$, verifica-se uma boa concordância em termos de comportamento dessa propriedade mecânica.

A análise dos resultados de módulo em compressão mostram desvios pouco significativos e menores que os observados para os valores de resistência à compressão. Essa diferença pode ser explicada pela região da curva tensão/deformação em que os dados de módulo são extraídos. O módulo é medido na região elástica da curva, que é uma região linear de menores incertezas, que a região plástica, de onde se determinam os valores de resistência. Outro fator que pode influenciar nos resultados, colaborando com baixos desvios, é a excelente qualidade das matérias-primas empregadas na indústria aeronáutica e no rígido controle dos parâmetros de processamento, que resultam em materiais de boa qualidade e homogêneos, como constatado neste trabalho por meio da inspeção por ultrassom realizada nos laminados processados.

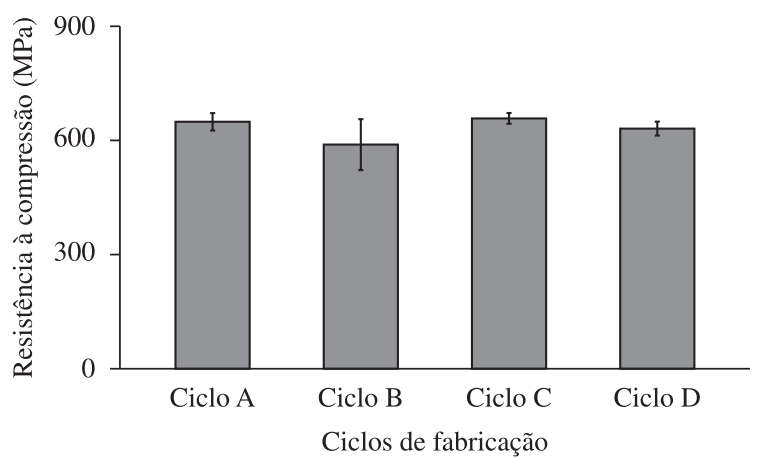

Figura 3. Resistência à compressão dos laminados de PPS/fibra de carbono obtidos sob diferentes ciclos de processamento.

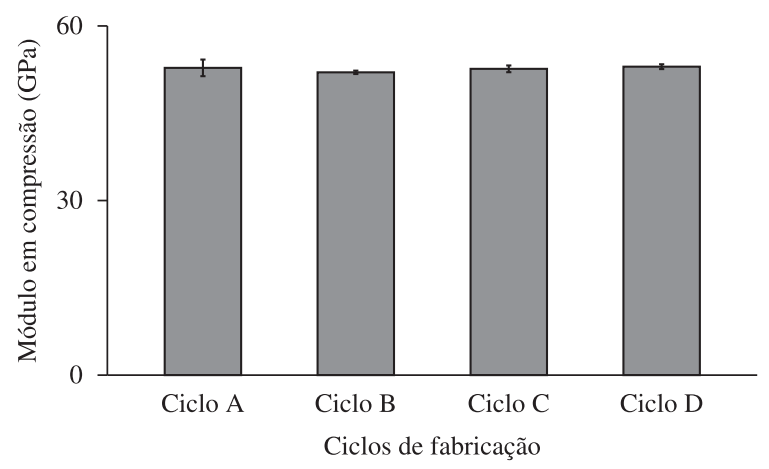

Figura 4. Módulo em compressão dos laminados de PPS/fibra de carbono obtidos sob diferentes ciclos de processamento. 
A seguir são apresentados os resultados dos ensaios de resistência e módulo em flexão, Figuras 5 e 6 respectivamente. Comparando-se os resultados de resistência da Figura 5 com valores disponíveis na literatura, referentes a laminados de PPS/ fibra de carbono obtidos por moldagem por compressão a quente ${ }^{[18]}$ (de 830 a $1039 \mathrm{MPa}$ para laminados ensaiados na direção $0^{\circ}$ e de 684,2 a 892,6 MPa para amostras ensaiadas na direção de $90^{\circ}$ ), verifica-se uma boa concordância entre os resultados. Boa concordância é também observada para os resultados de módulo em flexão obtidos na caracterização dos laminados processados em autoclave (Figura 6) e os disponíveis na literatura para compósitos de PPS/carbono obtidos por moldagem por compressão a quente (de 54,8 a 55,6 GPa na direção $0^{\circ}$ e de 43 a $45,1 \mathrm{GPa}$ na direção $\left.90^{\circ}\right)^{[18]}$. Os dados experimentais obtidos neste estudo também apresentam boa concordância com os fornecidos pelo fabricante (Tabela 1) ${ }^{[7]}$.

A correlação dos resultados dos ensaios de flexão com os dados de cristalinidade mostra que a família de laminado que apresenta a maior porcentagem de cristalinidade, favorecida

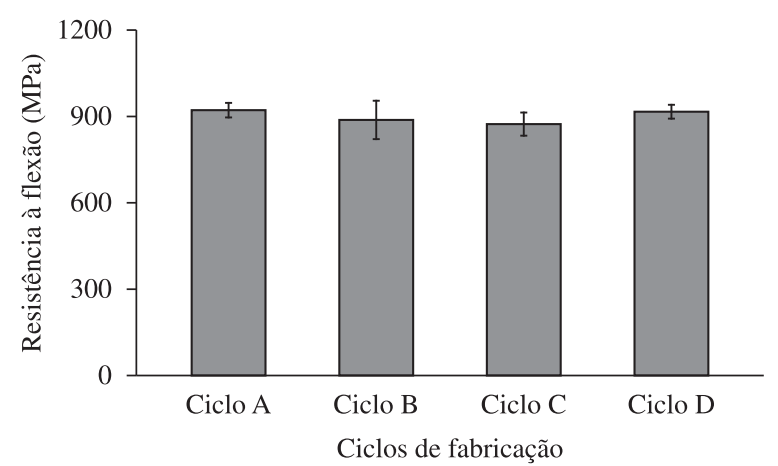

Figura 5. Resistência à flexão dos laminados de PPS/fibra de carbono obtidos sob diferentes ciclos de processamento.

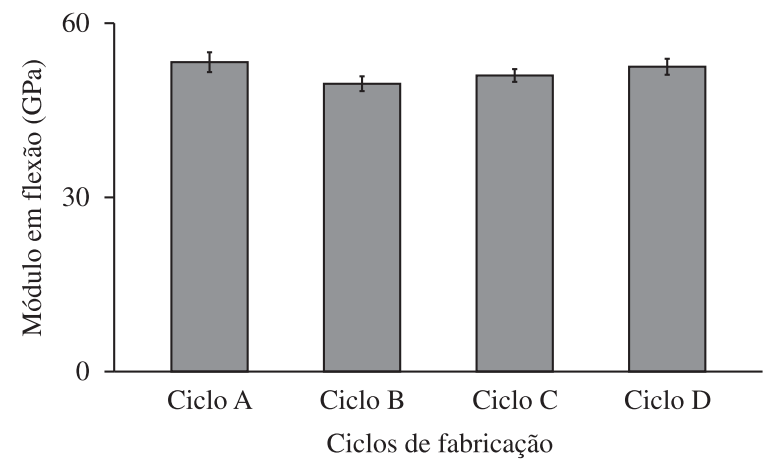

Figura 6. Módulo em flexão dos laminados de PPS/fibra de carbono obtidos sob diferentes ciclos de processamento.

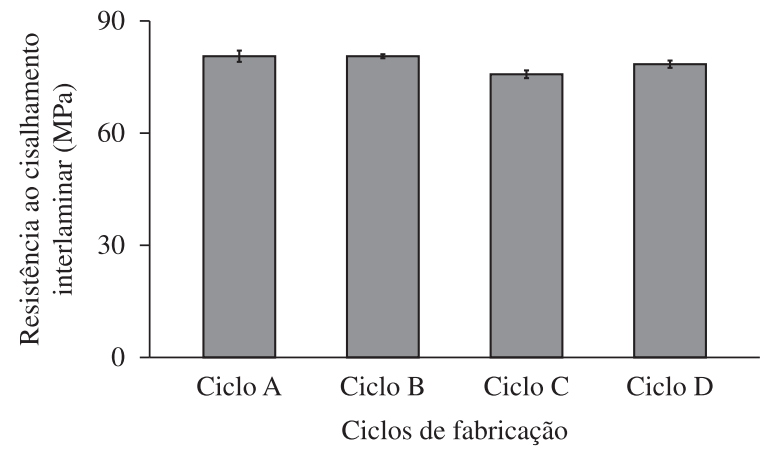

Figura 7. Resistência ao cisalhamento interlaminar dos laminados de PPS/ fibra de carbono obtidos sob diferentes ciclos de processamento. pela menor taxa de resfriamento, Ciclo $\mathrm{B}$, revela um ligeiro decréscimo nas propriedades de resistência e de módulo. Esse resultado obtido não é concordante com o trabalho de Deporter e Baird $^{[19]}$, onde laminados que tiveram o seu grau de cristalinidade aumentado de 10 para $46 \%$ apresentam propriedades em flexão significativamente maiores. Nesse estudo, os autores apresentam um aumento de $111 \%$ na resistência e $18 \%$ no módulo ${ }^{[19]}$. No entanto, esse comportamento vem sendo discutido, sabendo-se que as propriedades de flexão são influenciadas por modos de tração, compressão e cisalhamento $^{[6]}$.

Nesse sentido, um outro trabalho da literatura aponta que as resistências à flexão e à tração transversal são influenciadas por outros mecanismos, relacionados não somente com o grau de cristalinidade, mas também com aspectos morfológicos da matriz polimérica, como natureza e tamanho das estruturas cristalinas $^{[9]}$.

Apesar deste estudo não apresentar diferenças significativas nas resistências à flexão das diferentes famílias de laminados, em função das porcentagens de cristalinidades obtidas, sugere-se, como estudo futuro, análises dedicadas à avaliação detalhada da morfologia cristalina da matriz polimérica, de modo melhor elucidar a correlação desse parâmetro com o desempenho em flexão de laminados termoplásticos semicristalinos.

A Figura 7 apresenta os resultados referentes aos ensaios de cisalhamento interlaminar dos laminados de PPS/fibra de carbono processados. Nesses ensaios observa-se, apesar de pouco significativo, um ligeiro decréscimo na resistência ao cisalhamento interlaminar do laminado obtido pelo ciclo $\mathrm{C}$, com uma redução de aproximadamente $5 \%$ da resistência em relação aos laminados processados pelos ciclos A e B. Analisando-se os parâmetros do ciclo $\mathrm{C}$ de fabricação (Tabela 2), verifica-se que esse é o único que tem a aplicação de pressão entre 400 e $600 \mathrm{kPa}$ atuante durante o aquecimento. Esta queda da propriedade determinada pode estar relacionada à uma maior movimentação do reforço durante a fusão da matriz, com a aplicação da pressão, que pode ter afetado o alinhamento do reforço e/ou a formação da interface reforço-matriz, diminuindo a resistência ao cisalhamento interlaminar.

\section{Conclusões}

Os resultados de inspeção por ultrassom, análises térmicas e ensaios mecânicos de compressão, flexão e cisalhamento interlaminar obtidos neste estudo permitem concluir que a consolidação do compósito termoplástico de PPS/fibra de carbono em autoclave é viável. As imagens da inspeção por ultrassom de laminados obtidos em quatro diferentes ciclos de consolidação mostram que esses se apresentam homogêneos e sem a presença de descontinuidades. Análises de DSC revelam que as porcentagens de cristalinidade dos laminados variam entre $18,1-29,7 \%$, sendo que o ciclo com menor taxa de resfriamento favoreceu a maior cristalinidade da matriz polimérica. Os resultados dos ensaios mecânicos realizados mostram valores equivalentes aos apresentados na literatura para laminados processados por moldagem por compressão a quente e, também, que as amostras com maior cristalinidade $(29,7 \%)$ apresentam a menor resistência à compressão.

\section{Agradecimentos}

Os autores agradecem os apoios recebidos do CNPq (Processo $\left.n^{\circ} 305478 / 2009-5\right)$, e das empresas TEN CATE e EMBRAER pelo fornecimento da matéria-prima e disponibilização da infra-estrutura, respectivamente, e da técnica química Andreza de Moura da Divisão de Materiais/IAE, pela realização das análises térmicas. 


\section{Referências Bibliográficas}

1. Botelho, E. C. \& Rezende, M. C. - Polímeros, 10, p.E4 (2000).

2. Armstrong, K. B.; Bevan, L. G. \& Cole, W. F. - "Care and Repair of Advanced Composites", SAE (2005).

3. Hou, M.; Ye, L.; Mai, L. W. - J. Mater. Proc. Technol., 63, p.334 (1997).

4. Botelho, E. C.; Costa, M. L.; Mazur, R. L. \& Rezende, M. C. Polímeros, 18, p.237 (2008).

5. Vodicka, R. - "Thermoplastics for Airframe Applications - A Review of the Properties and Repair Methods for Thermoplastic Composites", Defense Science and Technology Organization, Australia (2006).

6. Ten Cate Advanced Composites. - "CETEX Thermo-Lite TC1200 PEEK Resin System Technical Data”, Disponível em: <http:// www. tencate.com>.

7. Ten Cate Advanced Composites. "CETEX PPS resin system Technical Data”, Disponível em: <http:// www.tencate.com>.

8. Vieille, B.; Aucher, J. \& Taleb, L. - Mater. Sci. Eng., 517, p.51-60 (2009).

9. Spruiell, J. E. \& Janke C. J. - "A review of the measurement and development of crystallinity and its relation to properties in neat poly(phenylene sulfide) and its fiber reinforced composites", Department of Energy, USA (2004).

10. Ten Cate - “CETEX PPS Guide lines”, CETEX - ITD4401.01, Nijverdal (2001).
11. Pantelakis, S. G.; Katsiropoulos, C. V.; Labeas, G. N. \& Sibois, H. Compos. Part A, 40, p.595 (2009).

12. Fernandez, I.; Blas, F. \& Frovel, M. - J. Mater. Proc. Technol., 143-144, p.266 (2003).

13. Offringa, A.; List, J.; Teunissen, J. \& Wiersma, H. "Fiber Reinforced Thermoplastic Butt Joint Development”, in: SAMPE Conference 2008, Long Beach-CA, may (2008).

14. Meyer, D. R.; Bersee, H. E. N. \& Beukers, A. "Temperature effect on reinforced thermoplastic composite properties for primary aircraft structure applications", in: 49 ${ }^{\text {th }}$ AIAA/ASME/ASCE/AHS/ ASCStructures, Structural Dynamics, and Materials Conference, Schaumburg-IL, apr (2008).

15. Franco, L. A. L.; Graça, M. L. A. \& Silva, F. S. - Mater. Sci. Eng. A, 488, p.505 (2008).

16. Canevarolo, S. - "Técnicas de Caracterização de Polímeros”, Artliber, São Paulo (2007).

17. Ijaz, M.; Robinson, M. \& Gibson, A. G. - Compos. Part A, 38 p.828-842 (2007).

18. Costa, G. G. - "Avaliação da Influência dos Ciclos Térmicos nas Propriedades dos Compósitos Termoplásticos de PPS e PEI com Fibras de Carbono e Vidro Conformados por Prensagem a Quente", Tese de Mestrado, Instituto Tecnológico de Aeronáutica, Brasil (2006).

19. Deporter, J. \& Baird, D. G. - Polym. Compos., 14, p.201 (1993).

Enviado: 04/12/09

Aceito: $11 / 05 / 10$

DOI: $10.1590 / \mathrm{S} 0104-14282010005000049$ 